be solved for $s .^{*}$ This completes the proof of the theorem.

If $f(x)=0$ is of degree 7 or $8, \dagger$ there exist three $\phi$-functions having the properties of Brioschi's theorem. We then set up a transformation similar to (1), and the equations $\sum y^{3}=0, \sum y^{4}=0$ are homogeneous equations in three parameters. The determination of $c_{1}, c_{2}, c_{3}$ will, in general, lead to an equation of the 12 th degree, as was pointed out above.

If $f(x)=0$ is of degree 6 it does not seem possible to lower materially the maximum 24 which was obtained in the second paragraph.

The University of California at Los Angeles

\title{
SOME PROPERTIES OF MULTI-COHERENT CONTINUA
}

\author{
BY W. A. WILSON
}

1. In a recent paper§ C. Kuratowski gives the following definitions:

A continuum $C$ is called uni-coherent (or $n$-coherent) if for every decomposition of $C$ into two continua $K$ and $L$, where $C \neq K \neq L, K \cdot L$ has one (or $n$ ) components. A continuum $C$ is called multi-coherent, if $K \cdot L$ is not connected.

The properties of such continua are later developed in some detail. Among the theorems proved are the following.

* The very exceptional case in which the coefficients of $s^{4}, s^{3}, s^{2}, s$ all vanish, while the term free of $s$ does not vanish, may be handled by introducing homogeneous parameters in (3), that is, by putting $M=s+t$, $M_{2}=a_{2} s+b_{2} t$, etc. For the present case, $t$ must be zero.

$\dagger$ Although Brioschi's theorem does not mention equations of even degree, $(n-1) / 2 \phi$-functions can be set up for an equation of even degree $n+1$ just as they can for an equation of odd degree $n$, and in different ways as well.

$\ddagger$ Presented to the Society, October 27, 1928.

$\S$ C. Kuratowski, Sur la structure des frontières communes d deux regions, Fundamenta Mathematicae, vol. 12, pp. 20-42. 
(I) For a continuum $C$ to be multi-coherent it is necessary and sufficient that, for every sub-continuum $Q$ of $C, C-Q$ is connected.

(II) If $C$ is a bounded plane set which is the common frontier of two regions, $C$ is a multi-coherent continuum.

(III) $A$ bounded plane continuum which is decomposable and bi-coherent is the common frontier of two regions.

A careless reading of the above definitions may give the erroneous impression that a multi-coherent continuum is $n$-coherent for some value of $n$. The continuum given in Ex. 4(a) (loc. cit., p. 23) is multi-coherent, but not $n$ coherent, since $K \cdot L$ may have either two or three components according to the manner of decomposition of $C$. Furthermore, as Kuratowski points out, the continuum just cited shows that the converse of Theorem III is not true. The converse of Theorem II also is not valid, and hence the class of multicoherent plane continua is larger than that of regular frontiers.* Although the problem of finding necessary and sufficient intrinsic conditions for a bounded decomposable continuum to be a regular frontier has been solved, $\dagger$ less general conditions may at times be more convenient to use and the close relationship between multi-coherent continua and regular frontiers seems to the author to make it of interest to determine additional conditions under which the former class of continua have the same intrinsic properties as the latter.

Certain properties follow at once from the equivalent definition of multi-coherent continua given in Theorem I above. We first note that, if the bounded multi-coherent continuum $M$ is the union of two proper sub-continua $H$ and $K$, both $H$ and $K$ are irreducible about the set $H \cdot K$.

* A set is called a regular frontier if it is the frontier of at least two components of its complement.

† See C. Kuratowski, Sur la séparation d'ensembles situes sur le plan, Fundamenta Mathematicae, vol. 12, p. 235; and W. A. Wilson, On bounded regular frontiers in the plane, this Bulletin, vol. 34 (1928), p. 86. 
For otherwise either $H$ or $K$ would contain a sub-continuum disconnecting $M$. (A continuum $M$ is said to be disconnected by the set $C$ if $M-C$ is non-void and is not connected.) Furthermore, if $M$ is a bounded decomposable multi-coherent continuum, there is always an irreducible decomposition of $M$ into two proper sub-continua $H$ and $K .^{*}$ In this case no proper sub-set of $H \cdot K$ disconnects $M$ and we may say that $H \cdot K$ irreducibly disconnects $M$.

2. THEOREM. Let $M$ be a bounded multi-coherent continuum. Let $A$ and $B$ be proper sub-continua of $M$ which are not continua of condensation and let $A \cdot B=0$. Then $A+B$ disconnects $M$.

Proof. Set $K=M-(A+B)$. If $K$ is connected, $\bar{K}$ is a continuum. Now $A^{\prime}=A-A \cdot \bar{K}$ and $B^{\prime}=B-B \cdot \bar{K}$ are not void and $A \cdot B=0$, by hypothesis. Hence $A^{\prime}+B^{\prime}$ is not connected. But $A^{\prime}+B^{\prime}=M-\bar{K}$. Hence we have the contradiction that the continuum $\bar{K}$ disconnects $M$. Therefore $K$ is not connected.

3. Theorem. Let $M$ be a bounded multi-coherent continuum. Let $A$ and $B$ be proper sub-continua of $M$, let $A \cdot B=0$, and let $A+B$ disconnect $M$. Then $K=M-(A+B)$ has precisely two components.

Proof. Let $C$ be a sub-continuum of $M$ irreducible between $A$ and $B . \dagger$ Then $C-C \cdot(A+B)$ is connected and has limiting points on both $A$ and $B$. Hence at least one component $k$ of $K$ has limiting points on both $A$ and $B$ and $k+A+B$ is a continuum.

Since $A+B$ disconnects $M, K-k=M-(k+A+B)$ is not void. Since $M$ is disconnected by no sub-continuum, $K-k$ is connected. The theorem is proved.

4. THEOREM. Let $M$ be a bounded multi-coherent continuum. Let $A$ and $B$ be proper sub-continua of $M$ which are not continua

* This is shown in the reference at the beginning of this section, p. 25, Theorem $(\eta)$.

† This does not imply that $C$ contains either $A$ or $B$, but merely that $C \cdot A \neq 0 \neq C \cdot B$ and that there is no proper sub-continuum of $C$ having this property. 
of condensation and let $A \cdot B=0$. Let $K=M-(A+B)$. Then $\bar{K}$ is the sum of two continua which are irreducible between $A$ and $B$ and have no common points.

Proof. By $\S \S 2$ and $3, K$ has two components, $K^{\prime}$ and $K^{\prime \prime}$, one of which, say $K^{\prime}$, has limiting points on both $A$ and $B$. If $K^{\prime \prime}$ had limiting points on $A$ only, we would have the contradiction that $A$ disconnects $M$. Hence $\bar{K}^{\prime}$ and $\bar{K}^{\prime \prime}$ are continua joining $A$ and $B$ and $\bar{K}^{\prime} \cdot \bar{K}^{\prime \prime}=0$, since otherwise $\bar{K}^{\prime}+\bar{K}^{\prime \prime}$ would disconnect $M$.

Let $C$ and $D$ be sub-continua of $\bar{K}^{\prime}$ and $\bar{K}^{\prime \prime}$, respectively, irreducible between $A$ and $B$. Then $C \supset K^{\prime}$, for otherwise $C+A+B$ would disconnect $M$. Thus $C=\bar{K}^{\prime}$ and in like manner $D=\bar{K}^{\prime \prime}$, which proves the theorem.

Corollary 1. Let $M$ be a bounded multi-coherent continuum. Let $M$ contain two proper sub-continua which are not continua of condensation and have no common points. Then $M$ is the union of four continua $A, B, C$, and $D$, such that $A \cdot B=0$ $=C \cdot D, A$ and $B$ are irreducible between $C$ and $D$, and $C$ and $D$ are irreducible between. $A$ and $B$.

Proof. Let $A^{\prime}$ and $B^{\prime}$ be proper sub-continua of $M$ which are not continua of condensation and have no common points, and let $K=M-\left(A^{\prime}+B^{\prime}\right)$. Then $\bar{K}$ is the sum of two continua $C$ and $D$ which are irreducible between $A^{\prime}$ and $B^{\prime}$ and have no common points. Let $L=M-(C+D)$. Then $\bar{L}$ is the sum of two continua $A$ and $B$ which are irreducible between $C$ and $D$ and have no common points. It is evident that $A \subset A^{\prime}, B \subset B^{\prime}$, and $C$ and $D$ are irreducible between $A$ and $B$.

Corollary 2. If $M$ satisfies the hypotheses of Corollary 1 , it is the union of two continua $H$ and $K$ such that $H \cdot K$ is the sum of two closed sets between which both $H$ and $K$ are irreducible.

To see this set $H=A+C$ and $K=B+D$ in the above corollary.

Corollary 3. If $M$ satisfies the hypotheses of Corollary 1 
and is a plane continuum, it cuts the plane and is the frontier of exactly two components of its complement.

This conclusion follows from Corollary 2 and a theorem proved elsewhere.*

5. Theorem. Let $M$ be a bounded multi-coherent continuum. Let $A$ and $B$ be proper sub-continua of $M$, let $A \cdot B=0$, and let $A+B$ disconnect $M$. Let $L=M-(A+B)$. Then $\bar{L}$ is the union of two continua, which are irreducible between $A$ and $B$ and whose only common points lie on $A+B$.

Proof. Let $h$ and $k$ be the two components of $L$. Obviously $\bar{L}=\bar{h}+\bar{k}$. Set $H=\bar{h}$ and $K=\bar{k}$. As in the proof of $\S 4$ it can be shown that $H$ and $K$ are irreducible between $A$ and $B$. Since $h=H-H \cdot(A+B)$ and $k=K-K \cdot(A+B)$, and $h \cdot k=0$, it follows that $H \cdot K \subset A+B$.

Corollary 1. Let $M$ be a bounded multi-coherent continuum. Let $A$ and $B$ be continua of condensation of $M$, let $A \cdot B=0$, and let $A+B$ disconnect $M$. Then $M$ is the union of two continua $H$ and $K, H \cdot K=\alpha+\beta$, where $\alpha$ and $\beta$ are closed subsets of $A$ and $B$ respectively, and both $H$ and $K$ are irreducible between $\alpha$ and $\beta$.

COROLlary 2. In addition to the hypotheses of Corollary 1 let $M$ be a plane continuum. Then $M$ cuts the plane and is the frontier of exactly two components of its complement.

This follows from the reference under $\S 4$, p. 736 , Lemma IV.

6. Summary and General Discussion. The theorems and corollaries of $\S \S 4$ and 5 show that, if $M$ is a bounded multicoherent continuum which can be disconnected by the sum of two sub-continua, there are three possible cases: (1) when $M$ can be disconnected by the sum of two continua of condensation; (2) when $M$ can be disconnected by the sum of two sub-continua which are not continua of condensation;

* W. A. Wilson, On the separation of the plane by irreducible continua, this Bulletin, vol. 33, p. 739. 
(3) when of any two sub-continua disconnecting $M$, one is a continuum of condensation, but not the other. Cases (1) and (2) are not mutually exclusive; they have been treated in $\$ \$ 4$ and 5 and the results there obtained may be summarized as follows.

THEOREM. Let $M$ be a bounded multi-coherent continuum which can be disconnected by the sum of two sub-continua, both or neither of which are continua of condensation. Then $M$ is the union of two continua whose divisor is the sum of two closed sets between which both continua are irreducible.

CoROLlary. Let $M$ satisfy the hypotheses of the above theorem and lie in a plane. Then $M$ is the common frontier of exactly two of its complementary domains.

To make the third case definite, let $A$, but not $B$, be a continuum of condensation of $M$. If we use the notation of $\$ 5$, this means that $A \subset H+K$ and $B-B \cdot(H+K) \neq 0$. Also both $H$ and $K$ are indecomposable, for otherwise we would have Case (2). There are two possibilities: $H \cdot K \cdot B=0$ and $H \cdot K \cdot B \neq 0$. If $H \cdot K \cdot B=0, B$ contains a sub-continuum $C$ which is irreducible between $H$ and $K$ and may contain $B-B \cdot(H+K)$. If so, $M=C+H+K, C$ is indecomposable, and $C+H$ and $K$ are both irreducible between $C \cdot K$ and $H \cdot K$. On the other hand, this is not the only possibility, as the following example shows. Let $a, b, c, d, e$, and $f$ be six points. Let $H$ be irreducible between each pair of the points $a, b$, and $c$; let $K$ be irreducible between each pair of the points $a, d$, and $e$; let $C$ be irreducible between each pair of the points $b, d$, and $f$; let $D$ be irreducible between each pair of the points $c, e$, and $f$; let $B=C+D$; let $H \cdot K=a, C \cdot D=f$, $H \cdot B=b+c$, and $K \cdot B=d+e$; and let $M=H+K+B$. Then $M$ is multi-coherent and can be disconnected by the sum of the continua $a$ and $B$, but $M$ cannot be expressed as the union of two continua having two closed sets in common, between which both are irreducible.

Now suppose that $H \cdot K \cdot B \neq 0$. Set $L=B-B \cdot(H+K)$; then $L=M-(H+K)$ and is connected. Let $C$ be a sub- 
continuum of $B$ irreducible about $H \cdot K \cdot B$. Then $C \supset L$, as otherwise $C$ would disconnect $M$. Thus $M$ is the union of three continua, $H, K$, and $C$, where $H$ and $K$ are indecomposable and irreducible between $H \cdot K \cdot A$ and $H \cdot K \cdot B$, and $C$ is irreducible about $H \cdot K \cdot B$ and contained in $B$, but it can be proved that $M$ is not the union of two continua $P$ and $Q$ such that $P \cdot Q=\alpha+\beta$, where $\alpha \cdot \beta=0$ and $\alpha$ and $\beta$ are closed sets between which $P$ and $Q$ are irreducible.

That this case may exist is seen from the following example. Let $a, b, c$, and $d$ be four points in the $X Y$-plane in threespace; let $H$ be a continuum irreducible between each pair of the points $a, b$, and $c$; let $K$ be a continuum irreducible between each pair of the points $a, b$, and $d$; let $H$ and $K$ lie on opposite sides of the $X Y$-plane except for these four points; and let $C$ be a continuum lying in the $X Y$-plane and irreducible between each pair of the points $b, c$, and $d$, but not containing $a$. Then $M=H+K+C$ is multi-coherent and can be disconnected by the sum of the two continua $a$ and $C$; but it is not the union of two continua $P$ and $Q$ having the properties given in the last two lines of the previous paragraph.

7. Generalization of Previous Theorems. The properties of multi-coherent continua thus far obtained are readily extended. The following is the analog of $\$ 3$.

THEOREM. Let $n$ be an integer greater than 1 and let $M$ be a bounded multi-coherent continuum which cannot be disconnected by any set of $n-1$ or fewer sub-continua. Let $A_{1}, A_{2}, \cdots, A_{n}$ be proper sub-continua of $M$ and let $\sum_{1}^{n} A_{i}$ disconnect $M$. Then $K=M-\sum_{1}^{n} A_{i}$ has exactly two components.

Proof. The theorem has already been proved for $n=2$; let $n \geqq 3$. Now $M$ cannot be irreducible between each pair of the sets $\left\{A_{i}\right\}$, for then $K$ would be connected.* Let $P$ be a proper sub-continuum of $M$ irreducible between, say, $A_{1}$ and $A_{2}$; then $P-P \cdot\left(A_{1}+A_{2}\right)$ is connected and has

\footnotetext{
* For $M$ is irreducible between $A_{1}$ and $\sum_{2}^{n} A_{i}$ and the assumption that $K$ is not connected leads at once to a contradiction.
} 
limiting points on both $A_{1}$ and $A_{2}$. Thus for at least one component $k$ of $K, \bar{k} \cdot A_{1} \neq 0 \neq \bar{k} \cdot A_{2}$.

Suppose first that $\bar{k} \cdot A_{i} \neq 0$ for $r$ values of $i$, say for $i \leqq r<n$, and that $\bar{k} \cdot A_{i}=0$ for $r<i \leqq n$. Then $k+\sum_{1}^{r} A_{i}$ is a continuum not meeting $\sum_{r+1}^{n} A_{i}$, and

$$
K-k=M-\left[k+\sum_{1}^{r} A_{i}+\sum_{r+1}^{n} A_{i}\right]
$$

is not void and is connected by hypothesis, since the set in the brackets is the sum of $n-r+1$ continua. Let

$$
k^{\prime}=K-k+\sum_{r+1}^{n} A_{i} \text {. }
$$

Now

$$
M=k+k^{\prime}+\sum_{1}^{r} A_{i} ; \bar{k} \cdot k^{\prime}=\bar{k} \cdot(K-k)+\bar{k} \sum_{r+1}^{n} A_{i}=0 ;
$$

and

$$
k \cdot \bar{k}^{\prime}=k \cdot \overline{(K-k)}+k \cdot \sum_{r+1}^{n} A_{i}=0 .
$$

Hence $\sum_{1}^{r} A_{i}$ disconnects $M$, which is contrary to the hypo thesis.

Now let $\bar{k} \cdot A_{i} \neq 0$ for every $i$. Then $k+\sum_{1}^{n} A_{i}$ is a continuum and, as before, $K-k=M-\left(k+\sum_{1}^{n} A_{i}\right)$ is connected. This proves the theorem.

8. Theorem. Let $n \geqq 3$ and let $M$ be a bounded multicoherent continuum which cannot be disconnected by any set of $n-1$ or fewer sub-continua. Let $A_{1}, A_{2}, \cdots, A_{n}$ be proper sub-continua of $M$ and let $\sum_{1}^{n} A_{i}$ disconnect $M$. Then $M$ is the union of $\sum_{1}^{n} A_{i}$ and two indecomposable continua $H$ and $K$ such that $H \cdot K \subset \sum_{1}^{n} A_{i}$ and both $H$ and $K$ are irreducible between each pair of the continua $\left\{A_{i}\right\}$.

PRoof. By $\$ 7$ there are two components $h$ and $k$ of $M-\sum_{1}^{n} A_{i}$. Set $H=\bar{h}$ and $K=\bar{k}$. Then $H \cdot A_{i}$ and $K \cdot A_{i}$ are not void for any value of $i$.

Let $P$ be a sub-continuum of $H$ irreducible between $A_{1}$ and $A_{2}$, for example. If $P \cdot A_{i}=0$ for any $i$, say $i=3$, we have a contradiction, for then $\left(A_{1}+P+A_{2}\right)+A_{3}+\sum_{4}^{n} A_{i}$ disconnects $M$ and contains not more than $n-1$ components, unless $P=H$, in which case $M$ is disconnected by $A_{1}+A_{2}$ $+\sum_{4}^{n} A_{i}$, again a contradiction.

Thus $P \cdot A_{i} \neq 0$ for every $i$. Now let $P^{\prime} \subset P$ and be irre- 
ducible between $A_{1}$ and $A_{3}$. By the above reasoning $P^{\prime} \cdot A_{2} \neq 0$. Hence $P^{\prime}=P$. Hence $P$ is irreducible between each pair of the continua $\left\{A_{i}\right\}$ and so is indecomposable.

Finally $P \supset h$, as otherwise $P+\sum_{1}^{n} A_{i}$ would disconnect $M$. Hence $P=H$ and the theorem is proved.

Corollary. An n-coherent bounded continuum can be disconnected by n sub-continua, but not by fewer.

9. Theorem. Let $n \geqq 3$ and let $M$ be a bounded multicoherent continuum which cannot be disconnected by any set of $n-1$ or fewer sub-continua. Let $A_{1}, A_{2}, \cdots, A_{n}$ be proper sub-continua of $M$, let $\sum_{1}^{n} A_{i}$ disconnect $M$, and let $H$ and $K$ be the two indecomposable sub-continua of $M$ which are irreducible between each pair of the continua $\left\{A_{i}\right\}$. If every $A_{i}$ is a continuum of condensation, $M=H+K$ and $H \cdot K=\sum_{1}^{n} \alpha_{i}$, where each $\alpha_{i}$ is a closed sub-set of $A_{i}$. Otherwise there is but one continuum $A_{i}$ which is not a continuum of condensation and $M$ may not be the union of two continua whose divisor is the sum of two closed sets between which both continua are irreducible.

Proof. If two of the continua $\left\{A_{i}\right\}$, say $A_{1}$ and $A_{2}$, were not continua of condensation of $M$, we would have a contradiction, since $H+K+\sum_{3}^{n} A_{i}$ would disconnect $M$. If every $A_{i}$ is a continuum of condensation, $M$ has the properties stated above by $\S 8$. If one of the continua $\left\{A_{i}\right\}$ is not a continuum of condensation, an example similar to that of $\$ 6$ shows the truth of the last statement of the theorem.

Corollary. Let $M$ satisfy the hypotheses of the above theorem and lie in a plane. If every $A_{i}$ is a continuum of condensation, $M$ is the common frontier of exactly $n$ components of its complement.

YALE UNIVERSITY 